\title{
Belphégor
}

\section{Hidden Codes of Love : The Materiality of the Category Romance Novel}

\section{An Goris}

\section{Q OpenEdition}

1 Journals

\section{Electronic version}

URL: http://journals.openedition.org/belphegor/616

DOI: 10.4000/belphegor.616

ISSN: 1499-7185

Publisher

LPCM

\section{Electronic reference}

An Goris, «Hidden Codes of Love : The Materiality of the Category Romance Novel », Belphégor [Online], 13-1 | 2015, Online since 09 May 2015, connection on 21 December 2020. URL : http:// journals.openedition.org/belphegor/616 ; DOI : https://doi.org/10.4000/belphegor.616

This text was automatically generated on 21 December 2020.

\section{(c) (i) (9)}

Belphégor est mis à disposition selon les termes de la Licence Creative Commons Attribution - Pas d'Utilisation Commerciale - Pas de Modification 4.0 International. 


\title{
Hidden Codes of Love : The Materiality of the Category Romance Novel
}

\author{
An Goris
}

1 For the last few decades romance has been the popular genre par excellence in the English-speaking world. With sales figures that average around $\$ 1.36$ billion a year, a readership of nearly 75 million people in the U.S. alone and a $13.4 \%$ share of the American consumer book market in 2011, the popular romance novel is by far the bestselling genre in America ("About the Romance Genre"). In 2010 a staggering 8,240 new romance titles were released in the U.S., and 469 of these novels became national or international bestsellers. Harlequin, the most important romance publisher in the world, "publishes over 110 titles a month in 31 languages in 111 international markets on six continents" ("About Harlequin"). On average, the company sells about 130 million books a year ("Over Ons"); since its inception in the mid-twentieth century an astounding total of over 6 billion popular romance novels have been sold by this publisher alone ("About Harlequin").

2 While these impressive numbers indisputably establish the widespread popularity of romance, the genre has been studied very sparsely. Even though the scholarly examination of popular culture has become a respectable and well-established academic pursuit, few scholars turn their critical gaze towards this most popular (and feminine) of genres. Studies of the popular romance novel are consequently few and far between, and within this relatively small body of work attention to the material aspects of the genre has been very limited. ${ }^{1}$ This despite the fact that the material conditions of popular novels are of major importance since they function as sites of intense debate concerning the status, meaning, identity and cultural placing of the books.

3 This lack of critical attention paid to the romance novel in general and its material characteristics in particular may be a consequence of the widespread cultural prejudice that all romance novels are essentially the same. Although academics are generally taught to be critical of cultural stereotypes, in the case of the popular romance novel the 
academy seems to overwhelmingly buy into - and frequently even be at the origin of - the ingrained stereotypes of conventionality, formula and simplicity that surround the genre. As a result, the popular romance genre is largely ignored by academics, who deem books that are supposedly all the same unworthy of their critical attention. Somewhat surprisingly, a similar mechanism plays out within the developing field of popular romance studies with regard to the genre's materiality. ${ }^{2}$ Underlying this disregard is, I believe, the tacit assumption that the romance novel's materiality, which even more than other aspects of the genre is imbued with stereotypes and conventions, is a relatively simplistic and straightforward aspect of the genre that is free of the interpretative complexities romance scholars now regularly (and, notably, against the cultural grain) uncover in the genre's texts.

In this paper I examine if this tacit assumption remains valid when the romance novel's materiality is subjected to in-depth analysis. This analysis focuses in particular on the format of the category romance - the most conventional kind of romance novel - to uncover the fact that the genre's materiality is fundamentally and functionally marked by tensions between conventionality and originality, pattern and deviation, simplicity and complexity. The double reading of the category romance novel's materiality that is developed in this article and the perhaps surprisingly intricate relation between this materiality and the reader/public that is uncovered not only offer an overdue and innovative discussion of the category romance's materiality, but also develop interesting wider perspectives for the study of the complex relation between genre and materiality in popular culture more generally.

\section{The Category Romance Novel}

This paper focuses on a particular kind of romance novel, namely the so-called "category romance". As the name implies, category romances are popular romance novels that are published in a category or series (also called line or imprint), which groups together similar types of romance stories. ${ }^{3}$ Examples of category romance lines include the Blaze series (featuring sensual romances), the Intrigue line (featuring romances with a suspense subplot) or the Medical Romance imprint (featuring romances set in a medical context). Series membership traditionally determines a large part of the category romance's identity, which is reflected in the prominent place the series imprint takes up on the category romance's front cover (see figure 1). The front cover is traditionally also dominated by the eye-catching image of a couple (often partially undressed) locked in a passionate embrace. This image, known within the romance community as the "clinch", has become an iconic visual marker for popular romance in our culture and renders the novel's generic identity unmistakable (McKnight-Trontz 17 ; Wendell and Tan 170). 
Figure 1
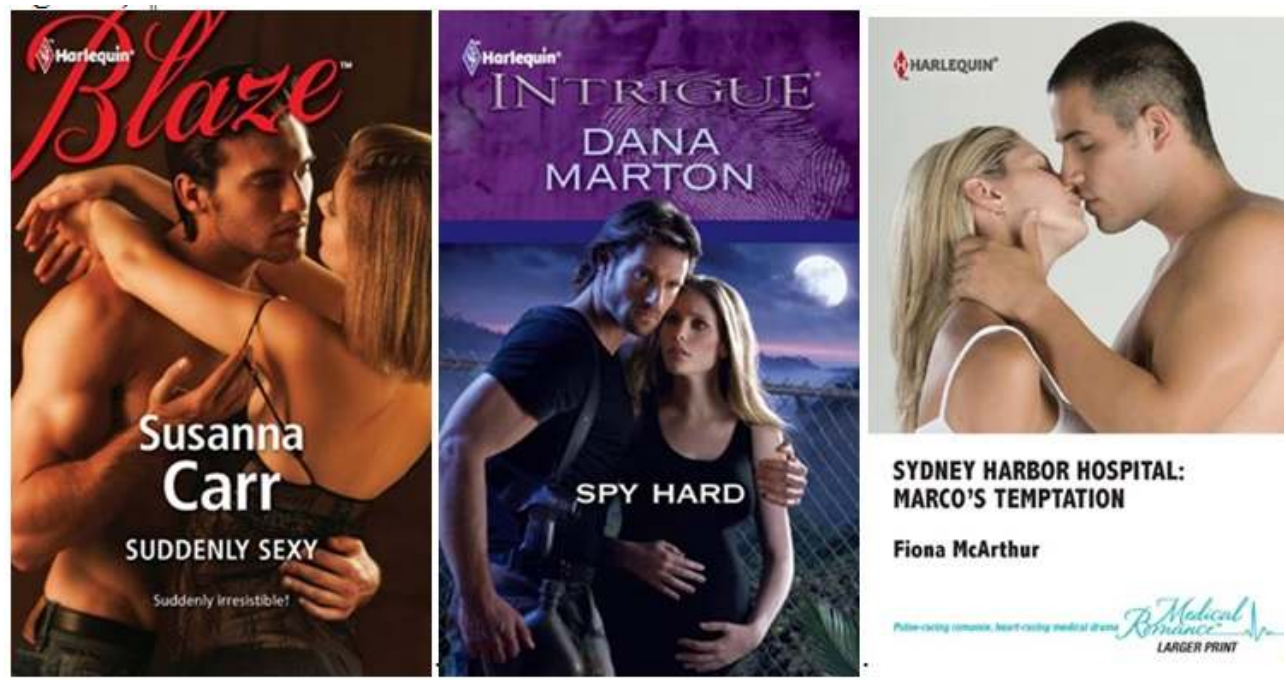

Category romances are published in the mass-market paperback format that is typically used for genre fiction in the English-speaking world. The category romance is an (in)famously cheap book that is available "wherever women shop" (as Harlequin's decades-old tagline boasts). This includes venues outside of the traditional literary circuit, such as supermarkets, grocery stores, newsstands and gas stations. Almost all category romances are published by the Canadian publisher Harlequin (or its subsidiaries Silhouette and Mills \& Boon), which releases over a hundred category romances each month in a wide variety of lines all over the world. ${ }^{4}$

The system of lines defines the category romance format in many ways and is perhaps more intricate than it seems at first sight. Each category romance is published in a series or line that has a particular narrative profile. Although these profiles appear to be characterized by a single defining trait (Blaze novels are erotic, Intrigue novels feature a suspense storyline, Medical Romance novels are set in a medical context), they are in fact composite and are made up of a conglomerate of narrative features. For example, Blaze novels are not only characterized by a high level of sensuality, but are also always set in a contemporary (usually North American) setting, feature a heroine who is between twenty-five and thirty-three and a hero between the ages of twenty-eight and thirtyeight and have an average word count of 60,000 words ("Harlequin Blaze"). ${ }^{5}$ Each line is thus differentiated from others via this conglomerate of primary and secondary linecharacteristics. Although the lines may appear simplistic to the outside world, the finelytuned differentiation between lines is very important within the genre's system, as the (commercial) viability of a line depends in part on the extent to which it can be differentiated from another line.

8 One of the consequences of this system is that category romances are highly conventional. All category romance novels share the overall conventions of the romance genre ${ }^{6}$ but within this encompassing generic framework each category romance also incorporates the conglomerate of conventions that characterize the line in which it is published. As a result, conventionality is pivotal to nearly every aspect of the category romance story and text. These conventions are, moreover, strongly enforced by editors and publishers, who act as gatekeepers guarding the genre and line profiles. Narratives that do not incorporate the various conventions are simply not published in the category 
romance system. ${ }^{7}$ The strong conventionality that consequently marks the category romance novel is often interpreted as a characteristic that renders the category romance an aesthetically inferior form, and it is frequently cited as one of the main reasons for the format's low cultural status. ${ }^{8}$

\title{
Materiality and the Category Romance Novel
}

9 Category romance novels are marked by a very typical look; their visual and material properties are instantly recognizable to almost everybody, romance readers and nonreaders alike. Indeed, regardless of our interest in or knowledge of the genre, most of us are able to instantly recognize a category romance novel when we see one. The question arises why the genre consistently chooses to adopt such material uniformity. What are the functions and effects of this remarkable semiotic strategy? That the visual and material design of a book is anything but a meaningless matter has been argued convincingly by the French theorist Gérard Genette. In his seminal study Paratexts he examines the role paratexts play in the reception and interpretation of a book. He argues that the paratext is

\begin{abstract}
a zone not only of transition but also transaction: a privileged place of pragmatics and strategy, of an influence on the public, an influence that - whether well or poorly understood and achieved - is at the service of a better reception of the text and a more pertinent reader of it (more pertinent, of course, in the eyes of the author and his allies). (2)
\end{abstract}

10 In other words, Genette suggests that the book's materiality has two main functions : it is a zone in which the book's producers and consumers negotiate the identity, meaning and interpretation of the text - a process that mainly consists of the producers' attempts to direct the consumers' reception of the text - and it serves to locate and reach the text's primary target audience. These functions are the paratext's main raison d'être ; indeed, as Genette remarks at the end of his study, functionality is "[t]he most essential of the paratext's properties" :

Whatever aesthetic intention may come into play as well, the main issue of the paratext is not to "look nice" around the text but rather to ensure for the text a destiny consistent with the author's purpose. To this end, the paratext provides a kind of canal lock between the ideal and relatively immutable identity of the text and the empirical (sociohistorical) reality of the text's public...the lock permitting the two to remain "level". (407-408)

11 Following this reasoning, analyses of the paratext should then not primarily focus on questions of aesthetics (is it beautiful or appealing?) but on questions of functionality (what purpose does it serve and which effects does it attempt to produce?).

This functionality should be understood in relation to the consumer of the paratext. In this regard Genette makes what to me appears to be a fundamental distinction between what he calls "the public" and "the reader":

[T] he public is not the totality of the sum of readers.... For a book...it seems to me that the public is nominally an entity more far-flung than the sum of its readers because that entity includes, sometimes in a very active way, people who do not necessarily read the book (or at least not in its entirety) but who participate in its dissemination and in its "reception".... The reader as conceived by the author...is, to the contrary,....a person who reads the text in toto.... The public as defined here, therefore, extends well and often actively beyond the sum total of readers. (74-75) 

image of a (scantily clad) man and woman locked in a passionate embrace. ${ }^{9}$ This image, known in the romance jargon as the "clinch", graces the front cover of an endless series of category romance novels in always changing but essentially similar variations. It appears to be a very apt visual representation of what the romance genre is all about. Depicting a man and a woman in an intimate and often explicitly passionate clinching embrace (hence the name), the image is made up of visual renderings of the nuclear elements of the conventional romance narrative: the hero, the heroine and their romantic (sexualized) interactions with each other. In a way it also offers a visual representation of what is arguably the core fantasy offered by the genre, namely that of an overwhelming and passionate romantic love focused absolutely on one other. 


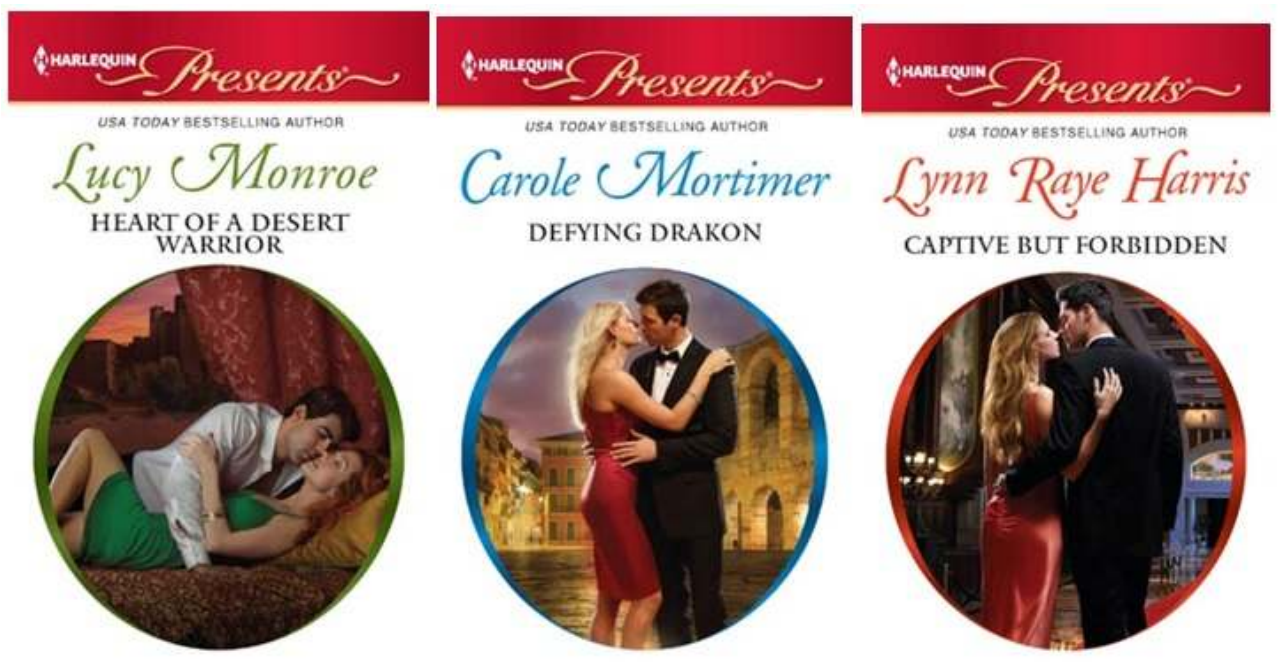

The clinch image carries a double semiotic code and has the potential to be interpreted differently by the public and the romance reader. To the public at large, the clinch image likely signifies simply the genre identity of the popular romance. This interpretation is based on the strong semantic connection between this image and the popular romance genre that exists in our culture as a result of the incessant reformulation of this type of image on the front cover of category romance novels since the 1970 s. ${ }^{10}$ As a visually striking and instantly recognizable image with only a limited range of potential variations, the clinch quickly attained an iconic status and has become the cover design shorthand par excellence for popular romance. Although the constant reformulations of the image on a seemingly endless string of category romance front covers reinforce and perpetuate a number of cultural stereotypes about the romance genre, including interpretations of the genre as formulaic, overly sexualized and more than a little ridiculous, the category romance is steadfast in its love for the clinch cover. ${ }^{11}$

of course the clinch image is, much like the narrative it so strikingly represents, a generic type. Each individual execution of the type is slightly different but essentially - typically the same. This typicality functions as the basis of the public's interpretation of the clinch image. That is, the public perceives the type of image and interprets this image as signaling a stereotypical kind of romance genre identity; this interpretation is based on the widespread cultural codes that regulate the semiotic functioning of cover iconography, which hold that a clinch image equals the generic identity "romance". In this interpretative act, the public overlooks the individual execution of this type - an execution that, for all its typicality, still has individual traits. These traits are, however, precisely the focal point of the romance reader's semiotic decoding of the image and suggest to her a somewhat different interpretation of the text's identity. They do not simply individuate the image, but do so according to a set of (generic) codes shared by the novel's producers and its target audience of romance readers. On the basis of these codes, the romance reader is able to learn more about the novel's specific characteristics.

19 Various elements of the clinch image are subjected to this genre-internal system of secondary codification. For example, the attire worn by the cover models can function as a code for the novel's subgenre. That models on the cover of historical category romances 
wear clothes that are instantly recognizable as period costumes comes as no big surprise and is a fairly obvious example of such a secondary codification of the clinch image. Other clothing-based codifications that are common in the romance genre are perhaps less obvious to the public at large. The relatively recent trend in paranormal romance to deck out cover models (particularly women) in leather clothing is probably not well-known outside the genre. Nonetheless, as romance bloggers Sarah Wendell and Candy Tan have observed, within the romance genre a female cover model dressed in leather or spandexlike clothing serves as a reliable semiotic sign that the novel in question features a paranormal storyline (178).

The relative state of dress or undress of the cover models is another coded element of the clinch. Although in the past this code seems to have been stricter than it is today, ${ }^{12}$ as a rule of thumb we can still assume that the more flesh is visible on the cover, the more sexually explicit the love scenes in the narrative are. The style in which the clinch image is drawn or photographed as well as the fashion and hairstyle worn by the cover models - all of which are cover elements that have been subjected to significant evolutions over the course of last three decades - are yet other coded parameters of the clinch that provide the experienced romance reader with additional information about the individual characteristics of the narrative - in this case, the date of publication. All of these parameters - time of publication, level of sensuality and subgenre - in fact serve to specify and singularize the text in the eyes of the romance reader. They function as important parameters of (narrative) differentiation within the romance genre's system and thus give the romance reader significant information about the particular qualities of the text.

21 The same clinch image is - or at least has the potential to be -interpreted rather differently by the public and the romance reader. Whereas to the former it likely functions as a code that signifies a very stereotypical, internally homogeneous kind of popular romance generic identity, to the latter the same image signals various kinds of specifications within this generic identity and essentially provides a message of generic heterogeneity. This semiotic mechanism of one material element simultaneously containing two codes (or two layers of codification) that mean different things to different consumers (the public versus the reader) is essential to the semiotic functioning of the category romance novel's materiality. It is a core principle that, as the rest of the analyses illustrate, underlies nearly every aspect of this materiality.

\section{Design Template}

One of the most visually striking and remarkable characteristics of the category romance's material packaging is the dominance of a standard design template that all novels published in the same line share. While each line or imprint has its own characteristic template, most of these templates also have certain elements in common (e.g. the spatial placing of certain elements). The line-imposed design template determines the look of nearly every aspect of the category romance's materiality and is as such quite an invasive material and visual presence. It is perhaps most noticeable on the front cover, where its dominant color scheme, prominent imprint logo and overall composition create an extreme sense of similarity between individual category romance novels published in the same line (see figures 2 and 3). As we can see in these examples, 
the placing and (stylistic) execution of almost every element of the cover composition (from the title to the name of the publisher) is determined by the line's design template.

Figure 3
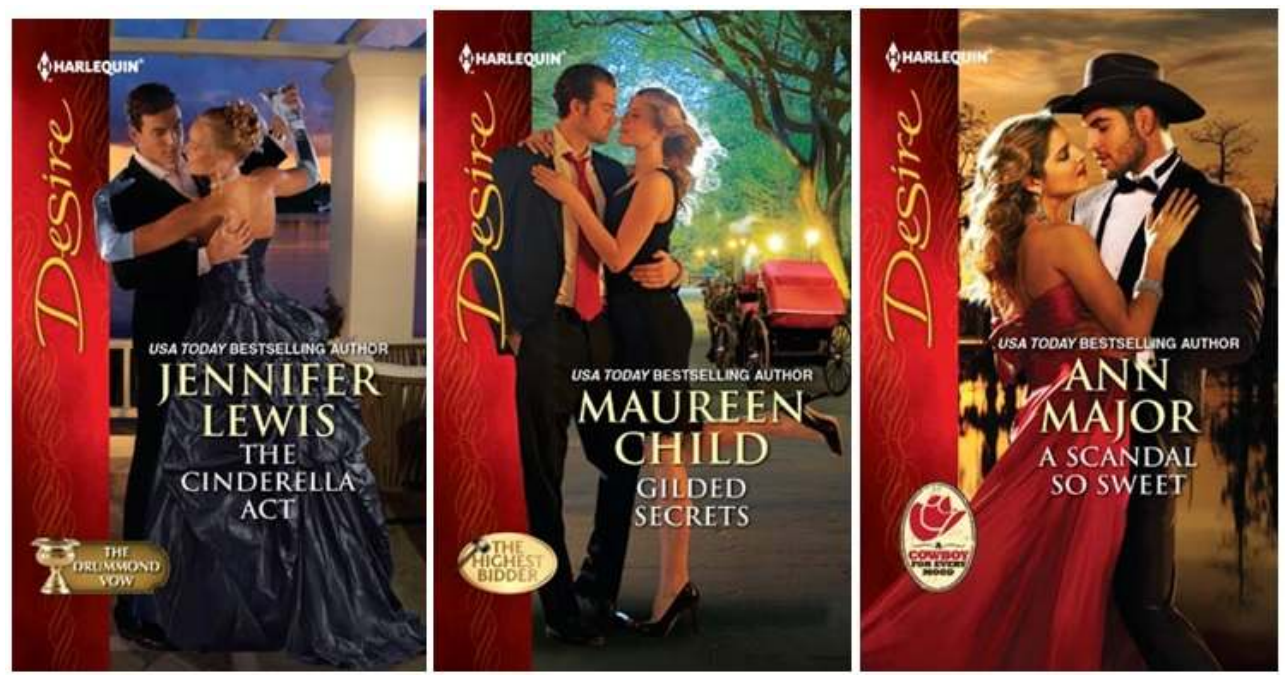

However, the impact of the line template extends far beyond the front cover. The back cover is designed in a very similar fashion, which makes for books that look altogether very similar (see figure 4). Indeed, as we can see in these examples, it is hardly possible to visually distinguish these individual books from each other. The imposing visual similarity seems to effectively stifle any claim to a more singularized interpretation that the book's back cover traditionally develops. The same compositional principle also applies to the materiality inside the book, which, much like its outside cover, is designed on the basis of the line template. Category romances published in the same line consequently share the same font, font size, composition of the title page, foreword, etc. In other words, they all look quite similar.

Figure 4
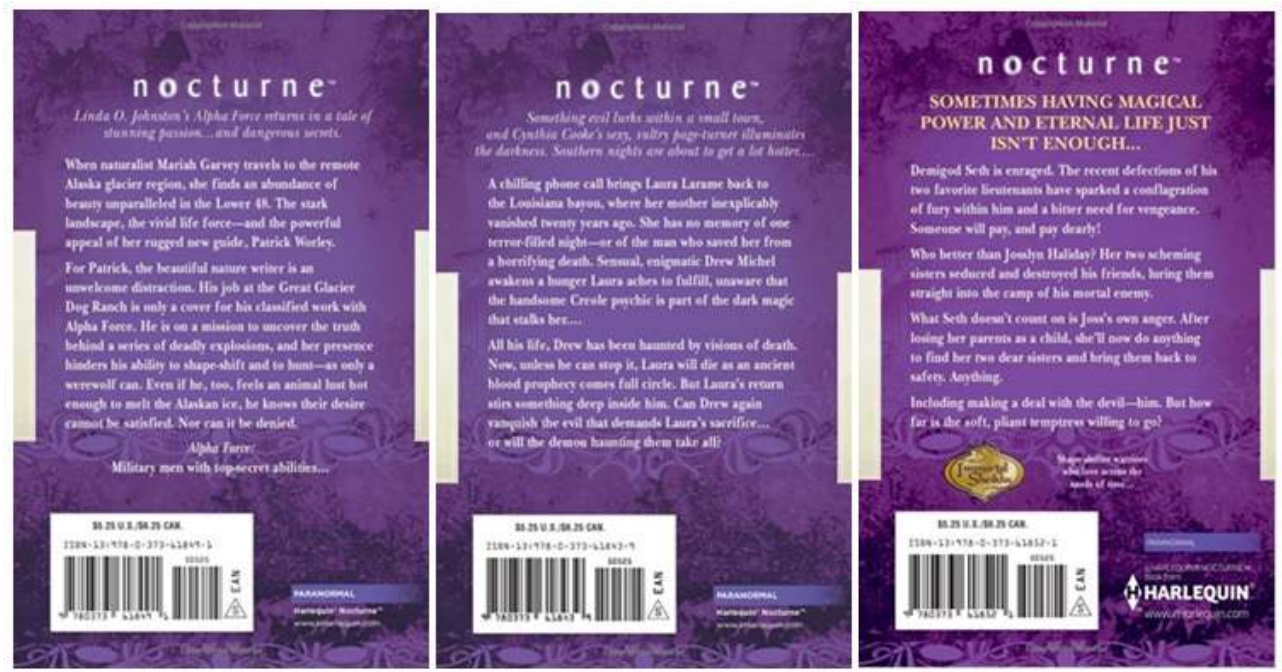

As the illustrations above show, the predominant effect of such an invasive design template is a very strong sense of standardization. It makes category romance novels look 
like uniform assembly-line products that lack the individuating and unique characteristics that we as a culture tend to value - particularly in art and literature. Instead, category romances all look the same and appear to be mutually interchangeable. This dominant impression of interchangeability is amplified by the fact that the elements that traditionally signify the book's individual identity, such as its title, the name of the author and the summarizing blurb on the back cover, are also slipped into the line's design template, which in effect almost completely mutes their message of idiosyncrasy. Instead, these elements appear to merely be (insignificant) variations upon an alreadyexisting pattern that in effect appears to be more meaningful. In the public's interpretation of the category romance's design template, this message of standardization is likely dominant and the line template in effect serves as a code signifying extensive similarity and lack of singularity.

Romance readers are, of course, not blind to the impression of similarity created by the line template, but they are nonetheless able to ascribe to it a different meaning. This interpretative difference is located in two aspects. First, whereas the public tends to connote the extensive visual and material similarity of the line template in a rather negative way (category romances are generally considered inferior forms of literature because they are - or at least materially appear to be - so similar), the category romance reader is inclined to interpret this similarity more positively as a code for the strong conventionality that is part and parcel of the category romance format and that its target reader appreciates. Second, the romance reader again, as in her reading of the clinch, detects a number of coded information parameters in the line template that in her eyes develop a more specific textual identity. For example, the template's dominant color scheme is often a code for the tone or subgenre of the line, as red signifies sensual lines, purple is conventionally reserved for suspenseful lines, white is typical of medical lines, etc. The line number printed on the spine of each category romance further reinforces the public's interpretation of the category romance novel as a standardized assembly-line product (Kamble 181), but to the romance reader this number denotes information about the line. The higher the number, the older the line is ; the older the line, the more likely it is to have proven its (commercial) success, but, by the same token, the more risk it runs of being outdated in the fast-changing, trend-sensitive popular romance genre.

The line name is yet another element that is interpreted differently by the public and the romance reader. While to the public the name denotes a simple narrative profile, for the romance reader familiar with the romance genre's refined system of lines it functions as a code for a more complex and composite narrative profile (cf. supra). This difference in interpretation or codification of the line name is particularly important in lines that appear to be quite similar. For example, Harlequin currently publishes two sensual lines, Harlequin Blaze and Harlequin Desire. The public is presumably unable to differentiate between these two lines since the names suggest similar erotic profiles, but the experienced romance reader is aware of the differences in the lines' respective compound narrative profiles. ${ }^{13}$ Whereas the public interprets these names as a codification of similarity, the romance reader interprets the line names as a codification of difference.

\section{The Preview Scene}

This brings me to what I call the preview scene, the third and final example of the double semiotic codification of the category romance's materiality. The preview scene is a scene 
(or more often part of a scene) printed on the first page of the category romance novel. It is a brief piece of text (usually about five to ten lines) that is placed before the title page, colophon, foreword or any other kind of introductory page. Following immediately on the book's front cover, the preview scene is usually the first page of the book the reader encounters. It consists of a partial scene that is extracted from the main narrative and that functions somewhat like a trailer for a film. Although the scene is presented without any explicit framing, its strategic placement at the beginning of the book sets up a metonymic interpretation of the scene as representative of the narrative from which it is extracted. The scene then functions to provide a first, very brief but supposedly representative taste of the narrative that follows a few pages later in the same volume.

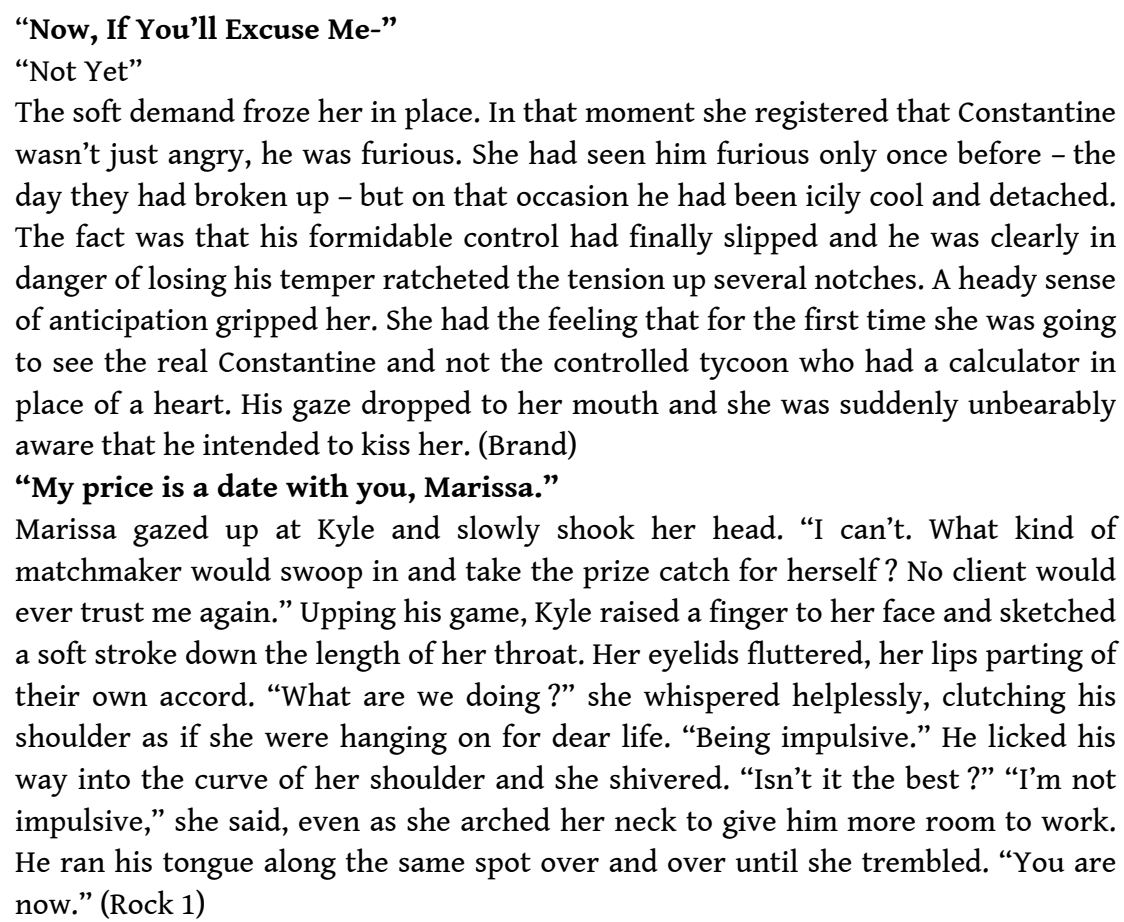

As we can see in these examples, these scenes are marked by a strong sense of conventionality. This is no coincidence; indeed, scenes that are selected as preview scenes usually depict a narrative moment that is instantly recognizable as a conventional part of a traditional romance plot. These scenes frequently zoom in onfeelings of sexual attraction and/or romantic conflict between the protagonists and depict events such as the erotically charged moments preceding the characters' first kiss or their first time making love. Invariably ending on an (erotic) cliffhanger, the preview scene is often a kind of narrative equivalent of the clinch image and is charged with the same sense of expectation, (sexual) tension and narrative determinism that marks the clinch. Like the clinch, the preview scene also represents a stereotypical image of the romance genre, not only because it depicts a very clichéd moment in the romance narrative, but also because this representation is rendered in a highly conventionalized, even hackneyed discourse.

Even more so than other material aspects of the romance, the preview scene is marked by a double codification and is accordingly interpreted rather differently by the public and the romance reader. In the public's interpretation, the extreme conventionality of the scene is the dominant feature and the scene is consequently interpreted as yet another code that signifies the novel's popular romance identity. Because the scene explicitly evokes stereotypes of the genre that are particularly widespread in our culture - the first 
kiss, the typical tension between conflict and attraction that is widely associated with popular romances, the clichéd and euphemistic language describing sexual attraction, etc. - this interpretation is guaranteed irrespective of the reader's profile. Indeed, even a reader who is only aware of the most basic cultural stereotypes surrounding popular romance recognizes in this scene the genre's conventions and will correctly interpret it as a code for the narrative's popular romance genre identity. In this process the preview scene not only invokes but also reinforces and perpetuates a number of the stereotypes already surrounding the genre, much like the clinch that is its visual equivalent.

Although the romance reader is obviously aware of the scene's strong conventionality and, like the public, interprets it as another element inscribing the novel in the popular romance genre, as a member of the romance genre's interpretative community she also has the ability to develop a different interpretation of this scene. In fact, when the romance reader reads this scene as a romance reader - that is, using the interpretative strategies particular to the genre - she is able to gain crucial new knowledge about the text and its specific, individual poetic properties. This is due to the fact that in the eyes of the experienced category romance reader the preview scene functions as a conceptual prefiguration of the creative interplay between conventionality and variation that is pivotal to the category romance's poetic functioning. This creative dynamic goes unnoticed by the public (and most of the genre's critics) because of their one-dimensional assessment of the genre's strong conventionality as only creating a pervasive sense of repetition and similarity between individual romance texts. However, this interpretation of conventionality fails to recognize how the web of conventions also creates a context in which every minute variation upon the convention stands out. ${ }^{14}$ This kind of variation - the brief deviation from the norm, the minor adaptation of the convention - represents a fundamental pillar of the category romance's poetic functioning and of the aesthetic pleasure the romance offers its readers. This particular creative dynamic is prefigured in the strongly conventional preview scene, which illustrates for the romance reader precisely how the author deals with the central creative task of the category format of fusing various sets of conventions with the appropriate amount of creative variation. Since a thorough knowledge of the genre's (and the line's) conventions is necessary to develop this interpretation, only generically initiated romance readers pick up on this dynamic and read the preview scene as something other than a pure reconfirmation of the novel's clichéd generic identity.

31 In a similar vein, the preview scene also allows the romance reader to get a first sense of what in the romance community is often referred to as the author's voice. In this context, the term "voice" refers to the conglomerate of elements that characterize an individual's writing (Goris, "Loving by the Book" 80). Voice is determined by both narrative and linguistic elements and includes such things as the rhythm of the text, the cadence of the dialogue, the pace of the story, the tone of the narrative, the development of the characters, etc. As I have pointed out elsewhere, voice is an important evaluation criterion in the popular romance genre and one that both editors and readers frequently cite as a potentially decisive factor in their evaluation of a particular romance novel. ${ }^{15}$ Although voice is a fluid and compound notion, the preview scene provides the romance reader with a first impression of the author's voice, which in many ways functions as the primary parameter of singularity in the category romance novel. Given the importance of the voice in the reader's enjoyment of the narrative, this impression is likely to factor 
into the reader's decision to read (and buy) the novel, and it thus bestows a commercially important function on the preview scene.

These latter two interpretations of the preview scene require extensive familiarity with creative dynamics and codes that are specific to the category romance format and are hence only developed by readers initiated in the popular romance genre - in other words, the category romance's target audience. For these readers, the preview scene functions not only as a code signifying the novel's romance generic identity (as it does to the public) but also as a codification of the novel's singularity. As with the clinch and the design template, these different interpretations of the category romance's materiality are also fundamentally tied to the public's and the reader's respective degrees of knowledge of and experience with the particular codes of the popular romance genre. The romance reader is able to develop a more complex and layered interpretation of the category romance's materiality than the public because her extensive knowledge of the genre's conventions and codes enables her to see differentiation where the public only sees similarity.

\section{Functions and Effects}

These exemplary analyses of three aspects of the category romance's material packaging indicate the systematic manner in which a double codification of this materiality is created. The potential for a double interpretation is a semiotic pattern that is present in nearly every aspect of these material conditions and that is implemented in a coherent and coordinated way. This suggests that far from being a random or coincidental effect, this semiotic pattern is a deliberate strategy on the part of the category romance novel's producers, who seek to influence the reception and interpretation of the text.

The stereotype-driven character of the category romance's materiality ensures that the public is likely to follow the producers' primary suggestion and interpret the book in generic terms as a popular romance novel. This interpretation is achieved via the repetitive material invocation of numerous widespread stereotypes that surround the genre in our culture. Although this strategy reinforces and perpetuates a very clichéd cultural image of the genre, one of its major interpretative benefits is that such stereotypes can be interpreted - decoded - by a huge and diversified audience. This public intelligibility of its material code is an important commercial consideration for a book that circulates in a wide variety of cultural and commercial spaces frequented by a wide variety of consumers.

That the public codification of the category romance novel's materiality revolves around a generic interpretation is no surprise given the commercial character of popular fiction. As scholars such as John Cawelti and Ken Gelder have argued, the field of popular fiction is much more preoccupied with the notion of genre than that of literary fiction:

One of the most productive ways to think about popular fiction is in terms of genre, a term that simply means - in our case - the type or species of fiction being written. The entire field of popular fiction is written for, marketed and consumed generically : it provides the primary logic for popular fiction's means of production, formal and industrial identification and critical evaluation. (Gelder 40)

Genre is thus one of the most important organizational and interpretative pillars of the field of popular fiction, and it steers the mass communication that marks this field in the right direction. Given the industrial, commercial and communicative prominence of 
genre in popular fiction, it is in fact only logical that the category romance novel - one of the most (in)famous examples of genre fiction - emphasizes this generic identity in the public codification of its materiality. As noted in the above analyses, this is hardly a subtle process. On the contrary, the category romance is very obvious in its material genre performance. This generic blatancy is, as Gelder has pointed out, another characteristic element of popular fiction, in which "generic identities are always visible. This is how it differs from Literature. Popular fiction announces those [generic] identities loudly and unambiguously" (42). Although it is precisely this very emphatic generic conventionality that gives rise to the stereotypical interpretation of genre literature in general and the category romance novel in particular as a kind of literature that is repetitive, formulaic and inferior to literary fiction, this is the price that the category romance novel is apparently willing to pay in order to achieve the generic transparency that is the commercial bread and butter of popular fiction.

Such a manifest material performance of the novel's generic identity is functionally important not only to the vast public of non-readers, but also to the book's target audience of self-identified category romance readers. Like the public, the romance reader recognizes the stereotype-driven public code as signaling the romance generic identity. This generic identification of the novel triggers, as has been established by Janice Radway's seminal study of romance readers, a set of generic expectations on the part of the reader. When the text meets these generic expectations - as the strongly conventional, editorially carefully controlled category romance specifically aims to do - the reader is satisfied. This interplay between the creation of generic expectations, the fulfilling of these expectations and the resulting reader satisfaction is of vital commercial importance to the category romance novel, as it provides the core impetus for the reader to want to repeat the reading experience by reading - that is, buying - other category romance novels.

However, this ostensibly homogeneous generic identity is thoroughly complicated in what I have called the secondary codification of the category's materiality - a genrespecific code that only readers familiar with the genre detect and decipher. Via these coded elements, the category romance's materiality suggests a more refined and singular interpretation of its text that is essentially designed to indicate how the novel is different from its generic colleagues. This hidden layer of the semiotic code not only enables the romance reader to develop a secondary set of textual expectations, but also thoroughly complicates the homogeneous image of the genre that is painted in the primary (public) layer of the book's material codification. Instead of further supporting the stereotypebased public interpretation of generic standardization, this (hidden) secondary layer of the material code consistently signals ways in which the romance novel develops a more specific identity. As illustrated in the analyses above, the degree of specificity of this identity increases gradually. Whereas the front cover is often still concerned with suggesting shared identity traits, such as subgenre, level of sensuality and line identity, the first page inside the book resolutely focuses on the text's singularity by showcasing the manifestation of authorial voice in the narrative text. 


\title{
Conclusion
}

40

The apparent simplicity of the category romance novel's materiality conceals a complex semiotic system of double encoding. The strong conventionality that marks the material packaging of the novel functions in a complex way that defies the stereotypes of simplicity, formula and repetition that surround the genre even as, on its surface level, it reinforces and perpetuates these same stereotypes. Whereas the mass public relies on this stereotype-confirming surface level to simplistically interpret the book as a formulaic instance of genre fiction, understanding - decoding - the hidden complexities of the underlying secondary semiotic layer requires the romance reader's extensive knowledge of both the romance genre's overall conventions and those that are specific to the category romance format. Only on the basis of such generic knowledge can this seemingly overwhelming conventionality be perceived and recognized as markers of variation and deviation instead of repetition and similarity.

41 The semiotic craftiness of this strategy lies not simply in the duality of the codification, but in the subtlety of its secondary layer. The stereotype-laden surface layer is such a strong visual and material presence that the category romance novel in fact actively courts the clichéd interpretations that this surface codification gives rise to and effectively invites the public to interpret its generic identity in a stereotypical manner. The secondary layer remains hidden from view and can only be perceived by those who always-already know it is there. Although this strategy is a testimony to the predominance of genre classifications in popular fiction, it also raises new questions about the accuracy and effectiveness of such simplifying classifications.

Indeed, the core interpretative mechanism uncovered in the material analyses in this paper - the notion that generically initiated readers interpret conventionality differently from readers who are not familiar with the genre's codes and conventions - has the potential to shed new light on the broader discussion of the role of conventionality in the popular romance genre specifically and other kinds of genre fiction more generally. It stands to reason that the dynamics uncovered in the category romance's materiality also apply to the text this materiality encloses and represents. Indeed, if we consider this materiality to be a physical manifestation and performative representation of the identity and characteristic traits of the text (as I have implicitly done throughout this discussion), the implications for the role of conventionality in the category romance narrative are potentially far-reaching and call for a renewed examination of the poetic functioning of both romance and other kinds of popular fiction.

\section{BIBLIOGRAPHY}

\author{
“About Harlequin.” Harlequin.com. Web. 4 July 2012. \\ “About the Romance Genre." Romance Writers of America. Web. 19 April 2012.
}


Brand, Fiona. A Breathless Bride. Ontario : Harlequin, 2012. Print.

Cawelti, John. Adventure, Mystery and Romance : Formula Stories as Art and Popular Culture. Chicago : University of Chicago Press, 1976. Print.

Darbyshire, Peter. "Romancing the World : Harlequin Romances, the Capitalist Dream, and the Conquest of Europe and Asia." Studies in Popular Culture 23.1 (2000) : n. pag. Web. 4 July 2012.

De Geest, Dirk, and An Goris. "Constrained Writing, Creative Writing : The Case of Handbooks for Writing Romances.” Poetics Today 31.1 (2010) : 81-106. Print.

Flesch, Juliet. From Australia with Love: The History of Australian Popular Romance Novels. Fremantle : Curtin University Books, 2004. Print.

Gelder, Ken. Popular Fiction : The Logics and Practices of a Literary Field. London : Routledge, 2004.

Print.

Genette, Gérard. Paratexts : Thresholds of Interpretation. Trans. Jane E. Lewin. Cambridge : Cambridge University Press, 1997. Print.

Goris, An. "Loving by the Book : Voice and Romance Authorship." New Perspectives on the Popular Romance Novel. Ed. Sarah S.G. Frantz and Eric M. Selinger. Jefferson : McFarland, 2012. 73-83. Print.

Goris, An. "Matricide in Romance Scholarship ? Response to Pamela Regis' Keynote Address at the Second Annual Conference of the International Association for the Study of Popular Romance." Journal of Popular Romance Studies 2.1 (2011) : n. pag. Web. 4 July 2012.

Goris, An. "Romance the World Over." Global Cultures. Ed. Frank Salamone. Newcastle : Cambridge Scholars, 2009. 59-72. Print.

Grescoe, Paul. The Merchants of Venus : Inside Harlequin and the Empire of Romance. Vancouver : Rainforest, 1996. Print.

“Harlequin Blaze." Harlequin.com. Web. 4 July 2012.

Jensen, Margaret Ann. Love's \$weet Return : The Harlequin Story. Bowling Green : Bowling State University Popular Press, 1984. Print.

Kamble, Jayashree. Uncovering and Recovering the Popular Romance Novel. Diss. University of Minnesota, 2008. Print.

Kolb, Elene M. “Checking Out The Categories.” Publishers Weekly 13 Nov. 1981 : 41. Print.

Kolb, Elene M. “The Books You Judge By Their Covers.” Publishers Weekly 13 Nov. 1981 : 42, 44, 51. Print.

Little, Jane. “Authorial Voice : The Many Hued Definitions.” Dear Author. 26 October 2010. Web. 4 July 2012.

McAleer, Joseph. Passion's Fortune : The Story of Mills \& Boon. Oxford : Oxford University Press, 1999. Print.

McKnight-Trontz, Jennifer. The Look of Love: The Art of the Popular Romance Novel. Princeton : Princeton Architectural Press, 2002. Print.

Mussel, Kay. “Where's Love Gone ?" Paradoxa : Studies in World Literary Genres 3.1-2 (1997) : 3-14. Print.

“Over Ons.” Harlequin.nl. Web. 4 July 2012. 
Paizis, George. "Category Romance in the Era of Globalization : The Story of Harlequin." The Global Literary Field. Ed. Anna Guttman, Michel Hockx, and George Paizis. Newcastle : Cambridge Scholars, 2006. 126-151. Print.

Radway, Janice. Reading the Romance : Women, Patriarchy and Popular Literature. Chapel Hill : University of North Carolina Press, 1984. Print.

Regis, Pamela. A Natural History of the Popular Romance Novel. Philadelphia : University of Pennsylvania Press, 2003. Print.

Regis, Pamela. What Do Critics Owe the Romance Novel ? Keynote Address at the Second Annual Conference of the International Association for the Study of Popular Romance." Journal of Popular Romance Studies 2.1 (2011) : n. pag. Web. 4 July 2012.

Roberts, Thomas. An Aesthetic of Junk Fiction. Athens : University of Georgia Press, 1990. Print.

Rock, Joanne. One Man's Rush. Ontario : Harlequin, 2012. Print.

Selinger, Eric Murphy, and Sarah S.G. Frantz. "Introduction." New Perspectives on the Popular Romance Novel. Ed. Sarah S.G. Frantz and Eric Murphy Selinger. Jefferson : McFarland, 2012. 1-20. Print.

Wendell, Sarah, and Candy Tan. Beyond Heaving Bosoms : The Smart Bitches' Guide to Romance Novels. New York : Fireside, 2009. Print.

Wirtén, Eva Hemmungs. "They Seek it Here, They Seek it There, They Seek it Everywhere : Looking for the "Global' Book." Canadian Journal of Communication 23.2 (1998) : n. pag. Web. 4 July 2012.

\section{NOTES}

1. The few studies of the popular romance novel that do consider the genre's material conditions are Juliet Flesch's From Australia with Love: The History of Australian Popular Romance Novels, Janice Radway's Reading the Romance: Women, Patriarchy and Popular Literature, Jayashree Kamble's Uncovering and Recovering the Popular Romance Novel and Jennifer McKnightTrontz's The Look of Love: The Art of the Popular Romance Novel.

2. Although academic work on the popular romance genre remains minimal in comparison to the academic work being done on other popular genres, a relatively small number of studies on the genre have been completed since the early 1980s. Academic attention has particularly increased over the last five years due to the establishment of the International Association for the Study of Popular Romance (IASPR) in 2009 and the launch of the academic peer-reviewed Journal of Popular Romance Studies in 2010. For more on these recent developments and an overview of the history of the burgeoning field of "popular romance studies," see Kay Mussel's "Where's Love Gone?," Pamela Regis' "What Do Critics Owe the Romance Novel?," An Goris' "Matricide in Romance Scholarship?" and Eric Murphy Selinger and Sarah S.G. Frantz's New Perspectives on the Popular Romance Novel.

3. Within the contemporary popular romance genre the category romance is differentiated from the so-called "single title romance", which is a longer romance novel that is not published in a series.

4. For more on the global distribution and consumption of category romances in different linguistic and cultural contexts, see Eva Hemmungs Wirtén's "They Seek it Here, They Seek it There, They Seek it Everywhere: Looking for the 'Global' Book," Peter Darbyshire, "Romancing the World: Harlequin Romances, the Capitalist Dream, and the Conquest of Europe and Asia," 
George Paizis' "Category Romance in the Era of Globalization: The Story of Harlequin" and An Goris' "Romance the World Over."

5. The precise narrative profile of a line is stipulated in the so-called "writing guidelines", which is a document for novice romance authors in which the publisher specifies the particular characteristics and conventions of a line. These writing guidelines are available on Harlequin's website.

6. Although it falls outside the scope of this paper to give a complete overview of the romance genre's conventions, the most important of these have to do with the basic plot of the romance narrative. An authoritative account of this plot is articulated by romance scholar Pamela Regis, who claims the romance narrative has eight conventional elements: "a definition of society, always corrupt, that the romance novel will reform; the meeting between the heroine and the hero; an account of their attraction for each other; the barrier between them; the point of ritual death; the recognition the fells the barrier; the declaration of heroine and hero that they love each other; and their betrothal" (Regis, A Natural History of the Popular Romance Novel 14).

7. For an account of how this particular system of publication might give rise to an interpretation of romance publishing as a form of constrained writing, see Dirk De Geest and An Goris' "Constrained Writing, Creative Writing: The Case of Handbooks for Writing Romances."

8. This negative appreciation of strong (generic) conventionality is something that affects (the cultural status of) all so-called genre fiction, but that is particularly strongly associated with the popular romance genre, which is, as Ken Gelder has noted, often considered the most conventional and conservative genre of popular fiction (43). For more on the connections between genre, conventionality and popular fiction, see John Cawelti's Adventure, Mystery, and Romance: Formula Stories as Art and Popular Culture (particularly the first two chapters).

9. While the clinch image is the most common image on the front cover of the category romance novel, other types of images include an image of a single person (most frequently a man) or a more domestic image of a couple with children or pets. For a (non-academic) discussion of these other types of romance front cover iconography, see Wendell and Tan 176-177.

10. Images of embracing couples have in fact been appearing on the covers of popular romance novels since British publisher Mills \& Boon developed the format in the 1930s and 1940s, so the semantic association between an embracing couple on the front cover and the generic identity romance is long-standing in our culture (McKnight-Trontz 40). Still, the more sexualized version of the embrace known as the clinch did not become commonplace in the popular romance genre until the 1970s, when the so-called "bodice ripper" romance started featuring more sensual embraces on the front cover (McKnight-Trontz 23-24).

11. It is interesting, however, that in Romance Writers of America's 2005 market study only $12 \%$ of romance readers indicated a preference for "romantic covers" while $35 \%$ indicated a preference for "sedate or abstract covers". The matter does not seem to be a potential deal breaker, however, as the majority of readers (53\%) indicated that they "prefer both types of covers".

12. In her 1984 study on the popular romance genre, Margaret Ann Jensen suggests that the category romance cover is composed according to a very strict code in order to indicate the narrative's level of sensuality: “The position of the hero and heroine on the books' cover is a good indication of how much sex there is in the romance. If they are not touching at all, the story does not have any sex scenes. If they are touching, the degree of sexuality escalates, with different touching positions symbolizing the amount of sexual involvement: "hands above waist=innocent frolic; hand below the waist or on the breast = sexual; prone positions=keep this in a locked drawer" (Kolb, "Checking Out The Categories" 41). The pictures also tell the readers how sexually responsive and aggressive the heroine is, as there are two types of embrace - the "hesitant heroine's" and the "cognizant heroine's": "The first kind is the more traditional portrayal of female sexuality and indicates that the hero pursues the heroine, who resists and 
perhaps capitulates, against her better judgment. The second kind of embrace is a departure from traditional sexual encounters and indicates that the heroine is responsive and probably even active in the pursuit of romantic-sexual gratification" (Kolb, "The Books You Judge By Their Covers" 62-63). Although Jensen's and Kolb's observations might have been accurate in the late 1970 s and early 1980s, category romance novel covers are no longer composed according to such strict rules. Still, the observation is indicative of the highly coded nature of the romance novel cover.

13. For the experienced romance reader the difference between these particular lines is in fact even more complex since the line that is now called Harlequin Desire used to be called Silhouette Desire and was published by Harlequin's subsidiary Silhouette. This subsidiary had a somewhat different profile than Harlequin itself, which was the result of the complex institutional history of the category romance market. Silhouette was originally founded in the early 1980 s as a separate publisher and one of the main competitors to Harlequin in the category romance market. This competition ended when Torstar, Harlequin's parent company, acquired Silhouette in 1984. Although from then on the two publishers essentially belonged to the same business conglomerate, Silhouette continued to be developed as a separate brand name with a somewhat more modern, progressive and specifically American profile than the Canadian Harlequin. Over time the differences between the two brands became less and less pronounced, and in April 2011 the Silhouette brand was discontinued and the imprints published under this brand, such as the Silhouette Desire line, underwent a slight name change. The distinction between Harlequin and Silhouette (or between such lines as Harlequin Desire and Harlequin Blaze) may seem insignificant to readers who are unfamiliar with the category market and its complex institutional history, yet it is highly significant to experienced romance readers, as is indicated by the fact that the two brands existed side by side within one publisher for twenty-five years. For more on this complex institutional history of the genre, see Paul Grescoe's The Merchants of Venus: Inside Harlequin and the Empire of Romance and Joseph McAleer's Passion's Fortune: The Story of Mills \& Boon.

14. This interpretation of the category romance's poetic functioning is in line with suggestions made by literary scholar Thomas Roberts, who compares the often misconceived aesthetic mechanisms underlying popular fiction (which he refers to as "vernacular fiction") to those at play in canonical poetry (such as the Spenserian stanza): “As suggested earlier, the pattern seems to play much the same role in vernacular fiction that the metrical scheme plays in a poem. In both cases, readers sense the formal scheme as the norm that permits them to appreciate the figural variations. The writers are like the jazz musicians who give us a familiar melody at the opening of the piece so that we understand the variations that follow. We do not listen for that melody. We listen for the variation" (165-166).

15. For more on the different definitions of voice that circulate in the romance community and the decisive role it plays in evaluations of romance novels by both readers and editors, see also Jane Little's “Authorial Voice: The Many Hued Definitions." 